\title{
Relative attention in judgments of heterogeneous similarity ${ }^{1}$
}

\author{
HANNES EISLER ${ }^{2}$ AND JAN KNÖPPEL \\ UNIVERSITY OF STOCKHOLM
}

\begin{abstract}
Shepard's (1964) study on similarity of stimuli with clearly discernible dimensions was repeated with some modifications, the most important being that the Os had to make numerical similarity estimates of the stimulus pairs. The overall outcome did not deviate much from Shepard's findings. By using quantitative estimates and choosing stimulus series so that the two dimensions were negatively correlated, data for each $O$ could be analyzed separately with a partial correlation technique. It was found that the more an $O$ attended to one dimension the less he attended to the other. The shifts in attention seemed to be random rather than regular. Consequently, the meaningfulness of a contention like Shepard's as to the nonexistence of a metric is questioned.
\end{abstract}

The present series of experiments was inspired by Shepard's (1964) study, "Attention and the metric structure of the stimulus space." In his experiment, Shepard used stimuli composed of two clearly discernible dimensions, viz, a circle of varying size with a radius of varying inclination. The Os were presented with a number of series of pairs of these stimuli; one stimulus of the pair was the same throughout the experiment and the other varied in a systematic way within each series, both in size of circle and inclincation of radius. (In a few series, one of the two variables was constant.) The Os were to point out for each series the pair exhibiting the greatest resemblance between the two stimuli.

From this experiment, Shepard concluded that no metric could fit group data, since most Os attended to only one of the two dimensions, i.e., either to circle size or to radius inclination. But Shepard argued that for single $O$ s under any one state of attention some Minkowski space would probably fit.

The present series of experiments
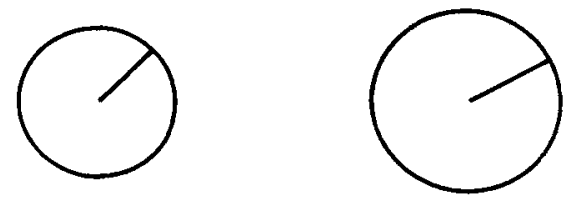

Fig. 1. One of the pairs of figures (Pair 9 H, see Fig. 2) used in Experiments 1 and 2 and in Shepard's experiments. constitutes a repetition of Shepard's study with the same stimulus material (a circular rim with a spoke, see Fig. 1). The aim was to study the distribution of attention between the two variables (circle size and angle) quantitatively for each $O$ separately.

\section{EXPERIMENTS}

The present study comprises two experiments: In Experiment 1 the Os were required to give a quantitative estimate of the similarity of every stimulus pair; Experiment 2 was a control experiment using the same forced-choice technique as Shepard.

Apart from the necessity of using similarity scales of at least ordinal level in order to be able to quantify the degree of attention, it was thought that the rating procedure might affect the consistency of choice of criterion in the Os. Moreover, a quantitative estimate allows for the possibility of an $\mathrm{O}$ reporting several pairs of a set as equally similar, and thus also for the possibility of the highest similarity being exhibited by more than one pair.

In Experiments 1 and 2, as well as in Shepard's experiment, each stimulus pair consisted of a standard stimulus and a comparison stimulus (Fig. 1). In Shepard's experiment, the stimulus pairs were divided into 24 series of 15 pairs each, with systematic variation of the two variables within each series (see Fig. 2). ${ }^{3}$

According to Shepard, his Os could, by and large, be divided into two groups: Os who took both dimensions into account in their choice of the most similar figure pair in a series and Os who took only one of the two dimensions into account, i.e., who chose that figure pair as most similar that matched the standard either in circle size or in inclination of spoke. From this point of view, Series $3,9,15$, and 21 are the most interesting because for them similarity regarding size of $\mathrm{rim}$ and inclination of spoke conflict most, and the distribution of choices for these four series is clearly bimodal. In these four series, two-dimension attenders would choose central figure pairs (G-I) as most similar, and matchers would choose Pairs C-E or K-M, depending on whether circle size or angle was matched and to which series the figure pair belonged. Only these four series were used in Experiments 1 and 2. In contrast to Shepard's experiment, no systematic variation in presenting the stimulus pairs was allowed. In Experiment 1, all stimulus pairs were randomized and in Experiment 2 each series was kept together, but the pairs within a series were randomized. Table 1 gives the design of Experiments 1-2 as well as the design of Shepard's experiment.

The rationale behind these modifications was to guide the Os as little as possible, in order to get spontaneous judgments. Shepard's (1964) and, perhaps still more, Hyman and Well's $(1967,1968)$ procedures may induce propensities in the Os to apply more or less conscious strategies.

\section{Experiment 1}

\section{STIMULUS MATERIAL}

In each figure pair, the standard stimulus was to the left and the comparison stimulus to the right. The arrangement is shown in Fig. 1. The four series $(3,9,15$, and 21 in Shepard's denotation) are made up of 60 figure pairs; since four comparison stimuli occur twice in the four series, only the 56 different figure pairs were used. These figure pairs were randomized and mimeographed in black with two pairs, i.e., four stimuli, in a row on five sheets with 11 or 12 figure pairs on each. The sheets were wired together in a different order for each $\mathrm{O}$ and constituted,

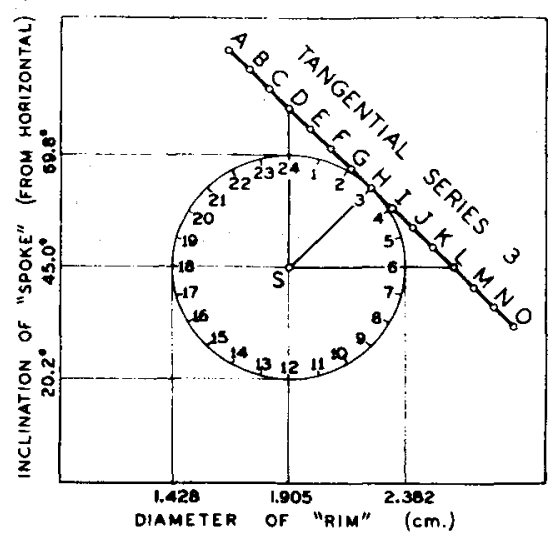

Fig. 2. "The physically defined space of the two-dimensional stimuli used in the present experiments, showing the manner and construction of the 24 tangential series of Exp. I." Figure and quotation from Shepard (1964), p. 61. The measure of the standard stimulus is illustrated graphically by the point " $S$ " in the center of the figure. 
Table 1

Comparison of Experiments 1 and 2 and Shepard's Experiment

\begin{tabular}{|c|c|c|c|}
\hline Condition & Experiment 1 & Experiment 2 & Shepard's Experiment \\
\hline $\begin{array}{l}\text { Number of stimulus } \\
\text { series (each consisting } \\
\text { of } 15 \text { figure pairs) }\end{array}$ & 4 & 4 & 24 \\
\hline $\begin{array}{l}\text { Order between figure } \\
\text { pairs when presented }\end{array}$ & $\begin{array}{l}\text { Randomized without } \\
\text { regard to stimulus } \\
\text { series }\end{array}$ & $\begin{array}{l}\text { Randomized within } \\
\text { each stimulus series; } \\
\text { order between series } \\
\text { randomized }\end{array}$ & $\begin{array}{l}\text { Regular within each } \\
\text { series; order between } \\
\text { series randomized }\end{array}$ \\
\hline Instruction & $\begin{array}{l}\text { Estimation of the } \\
\text { similarity of each } \\
\text { figure pair as a } \\
\text { percentage of identity }\end{array}$ & $\begin{array}{l}\text { Choice of the figure } \\
\text { pair in each series } \\
\text { exhibiting the great- } \\
\text { est similarity }\end{array}$ & $\begin{array}{l}\text { Choice of the figure } \\
\text { pair in each series } \\
\text { exhibiting the great- } \\
\text { est similarity }\end{array}$ \\
\hline Number of Os & 42 & 47 & 60 \\
\hline
\end{tabular}
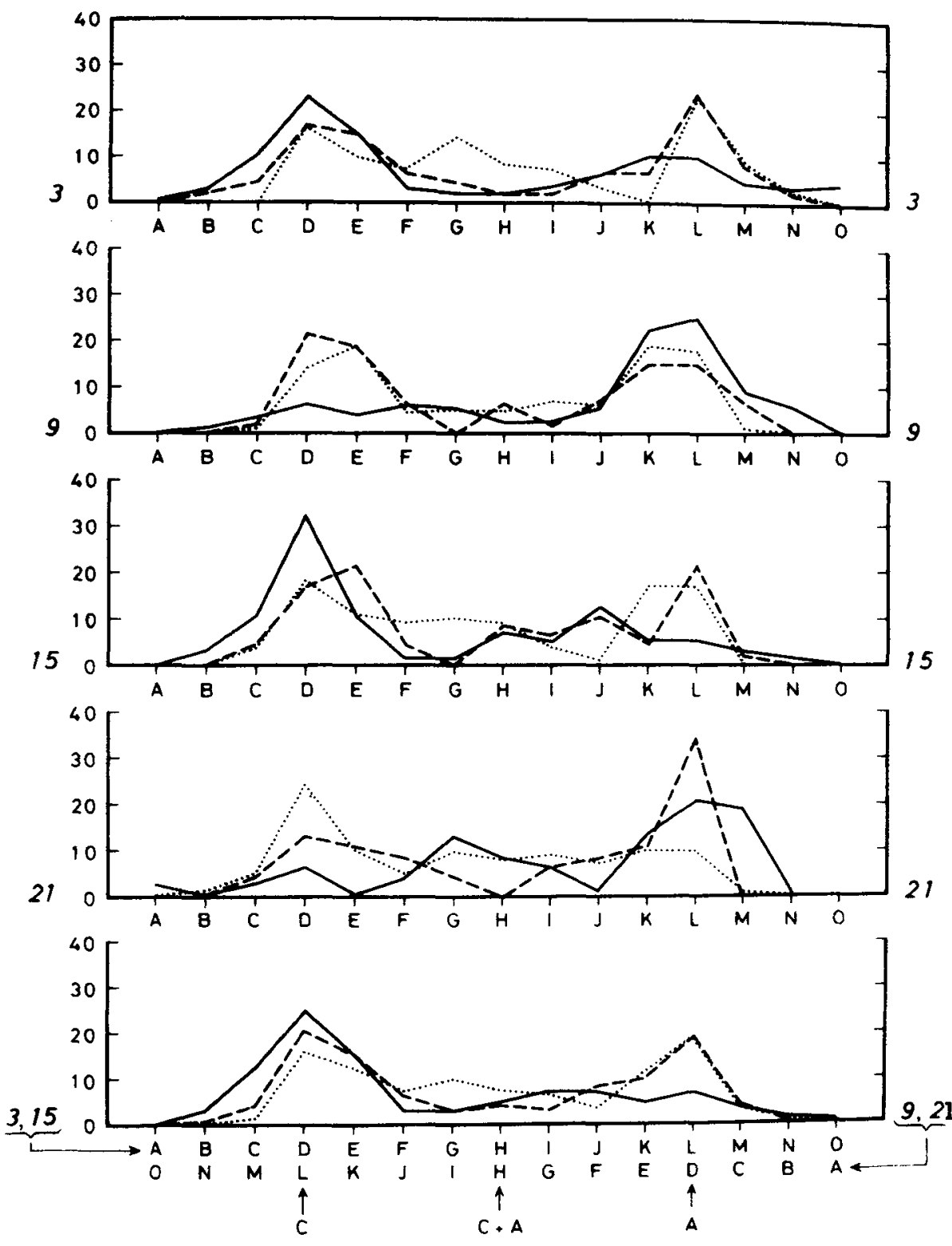

together with an instruction sheet, a small booklet.

\section{Experiment 2}

The stimulus material differed from the material in Experiment 1 in that the 15 figure pairs belonging to the same series were mimeographed on the same sheet (in random order) so that the booklets contained four pages. As a consequence, each of the four series was complete and thus 60 figure pairs were presented.

\section{PROCEDURE AND SUBJECTS}

\section{Experiment 1}

Having obtained the booklet, Os were required to estimate the similarity of each figure pair as a percentage of identity, i.e., on a scale between 0 (no similarity at all) and 100 (complete identity). The estimates were to be recorded on the pages between the two figures constituting a pair. The instruction was given both orally and in written form in the booklet. The experiment was carried out as a group experiment. Forty-four undergraduate students of psychology of both sexes participated; two of them had to be discarded because they had skipped a few figure pairs. Consequently, 42 Os supplied the data.

\section{Experiment 2}

The Os were required to circle the figure pair on each page that exhibited the greatest similarity. It was emphasized that every page should be studied thoroughly before deciding. The experiment was carried out as a group experiment. Forty-seven Os from the same population as in Experiment 1 participated.

\section{TREATMENT OF DATA AND RESULTS}

Choices of Greatest Similarity

To make the results of Experiment 1 comparable to Shepard's data, it was assumed that, for each series, the figure pair chosen by an $O$ as being most similar would be the one that obtained the highest similarity estimate in this series. If an $O$ assigned the highest similarity value to

Fig. 3. The upper four diagrams give the distributions in percent of the Os that chose each of the 15 figure pairs A-O for Series 3, 9, 15, and 21 , respectively. The lowermost diagram combines these four series so that choices for circle identity (C) and angle identity (A) coincide. Figure Pair $H$, marked by $(C+A)$, is the pair exhibiting the same similarity to circle size and angle according to Shepard (1964). Continuous line-Experiment 1; broken line-Experiment 2; dotted line-Shepard's experiment. 
Table 2

Percentage of Choices as Most Similar for Groups of Figure Pairs in Experiments 1 and 2 and Shepard's Experiment (Series 3, 9, 15, 21)

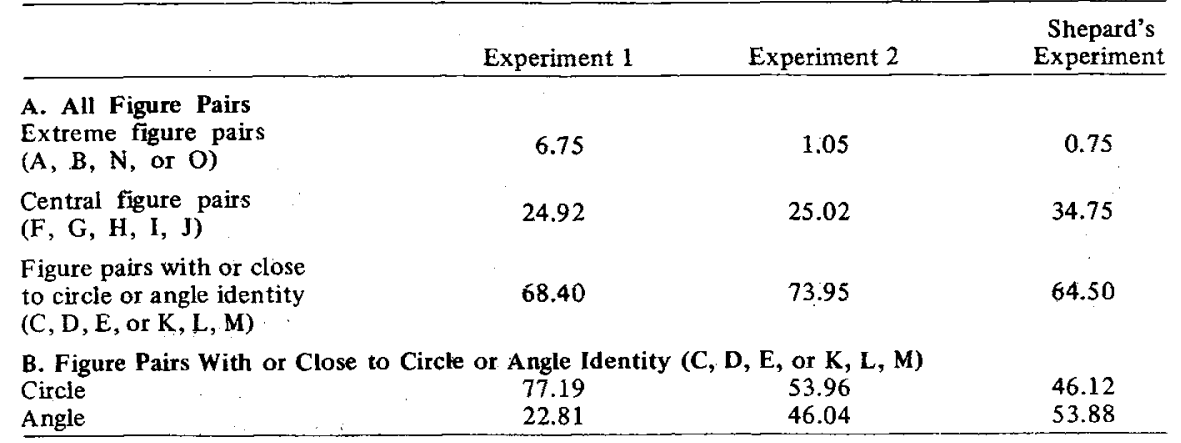

more than one figure pair in a series, his "choices" were divided between these pairs: if the highest similarity value was given to two pairs, the $O$ was considered to have picked each pair half a time; if it was given to three pairs, each pair obtained one-third choice, and so on.

For Experiment 2, the data treatment was straightforward: The number of choices of each figure pair was determined. The percentage of choices of each figure pair for Experiments 1 and 2 is given in Fig. 3 together with Shepard's results. Shepard's data were read off his Fig. 5 (Shepard, 1964), and are thus somewhat uncertain.

The results emerge perhaps more clearly from Table 2 than from Fig. 3. The following trends are found:

(1) Choices of extreme figure pairs. The frequency of choices of extreme figure pairs $(A, B, N, O)$ was greatest in Experiment 1.

(2) Comparison between choices of central figure pairs $1 O s^{\prime}$ taking both dimensions into account) and matching figure pairs $(O s$ taking only one of the dimensions, size or angle, into account). The ratio of the frequencies of choices of central figure pairs (G-I) to matching figure pairs (C-E and K-M) was greatest in Shepard's experiment.

(3) Comparison between choices of size-matching figure pairs and angle-matching figure pairs among choices

Table 3

$t$ Values (df $=3$ )

\begin{tabular}{|c|c|c|c|}
\hline $\begin{array}{c}\text { Variables } \\
(p=\text { relative frequency })\end{array}$ & $\begin{array}{l}\text { Experiment } 1- \\
\text { Experiment } 2\end{array}$ & $\begin{array}{l}\text { Experiment 1- } \\
\text { Shepard }\end{array}$ & $\begin{array}{c}\text { Experiment 2- } \\
\text { Shepard }\end{array}$ \\
\hline $\begin{array}{l}\text { Extreme Values: } \\
p_{A}+p_{B}+p_{N}+p_{O}\end{array}$ & $7.069 * *$ & $6.255^{* *}$ & 1.000 \\
\hline $\begin{array}{l}\text { Size Matching: } \\
p_{C}+p_{D}+p_{E} \text { for series } 3,15 \\
p_{K}+p_{L}+p_{M} \text { for series } 9,21\end{array}$ & $4.944^{*}$ & $7.824^{* *}$ & 1.951 \\
\hline $\begin{array}{l}\text { Angle Matching: } \\
p_{C}+p_{D}+p_{E} \text { for series } 9,21 \\
p_{K}+p_{L}+p_{M} \text { for series } 3,15\end{array}$ & $5.258^{*}$ & $4.310^{\star}$ & 0.146 \\
\hline
\end{tabular}

$* p<0.01 ; * p<0.05$
Discussion

To (1): Experiment 1 differs from the other two experiments in (a) the instruction (quantitative ratings of similarity), and (b) the complete randomization of the figure pairs. Probably both differences contributed to the result. The randomization hindered the Os from surveying a complete series, and the rating obliged the Os to judge each figure pair separately, both circumstances increasing the opportunity of giving occasional high ratings to extreme figure pairs. Keeping the series together and picking just one figure pair must have made "abnormal" choices more difficult.

To (2): Shepard's experiment differed from the other two experiments in (a) the total number of stimulus series presented, and (b) in the order of the figure pairs presented in each series. Point a may have entailed learning to take both stimulus dimensions into account, and Point b may have shown the Os more systematically that there is a variation in two dimensions. Both probably resulted in a tendency in relatively more Os to consider both dimensions in their choices, thus yielding a higher frequency for central figure pairs in the whole group. It should be noted that, because of the agreement on this point between Experiments 1 and 2, the instruction (rating vs choice) did not have any effect.

To (3): Experiment 1 differed from the other two in (a) instruction and (b) randomization between series. It is difficult to see how the randomization could have affected the proportion of Os attending to circle size compared to angle. Maybe quantitative estimation of sizes comes easier than that of angles, perhaps because of a habit of judging distances by size comparisons. In this case, the rating instruction is the cause.

In summary, complete randomization and forced attention to each figure pair separately seems to facilitate occasional abnormal judgments; systematic presentation of stimulus series and presentation of many such series with varying correlations between the two dimensions seems to entail a tendency to attend to both dimensions; and finally, quantitative rating seems to favor attention to size rather than to angle in one-dimension attenders.

Detailed Data Treatment of Experiment 1

Shepard implicitly assumed that the relation between the two stimuli in a dimension attended to by an $O$ in judging similarity was the difference, e.g., the difference between two circle diameters. He furthermore assumed that the spacing between adjacent stimulus values (A-B, 
Table 4

Correlations Between the Five Criteria Underlying Similarity Judgments. $(C=$ circle size, $A=$ angle,$D=$ difference, $R=$ ratio, $H=$ angle from horizontal, $V=$ angle from vertical.)

\begin{tabular}{lcrrr}
\hline & CR & AD & ARH & ARV \\
\hline CD & .98 & -.82 & -.80 & -.80 \\
CR & & -.83 & -.81 & -.81 \\
AD & & & .96 & .96 \\
ARH & & & & .84 \\
\hline
\end{tabular}
subjective magnitude. ${ }^{4}$ However, the ratio rather than the difference may be the pertinent relation (cf. Eisler, 1960). Therefore, we decided to use five criteria according to which the Os could have judged similarity, viz, difference between circle sizes, ratios between circle sizes, difference between angles, and ratios between angles, measured from the horizontal or the vertical. Since we were interested only in the rank orders of the figure pairs in accordance with these etc.) corresponded to equal changes in

criteria, considering the diameter or the area of the circles was of no consequence.

All figure pairs were ranked in accordance with the five criteria and all rank correlations were computed. Table 4 shows that the correlations between different criteria for the same dimension can be as low as .84.

The figure pairs were likewise ranked as to similarity estimates for every $O$ separately, and the rank correlations computed with all five criteria. Since the relation between physical values of the criteria for the figure pairs and the corresponding subjective values is unknown, apart from the very reasonable assumption of monotonicity, product moment correlations could not be used. [If the criterion is the difference, the use of rank correlations implies that the physical and subjective scales are related by a hypermonotone transformation (Suppes \& Zinnes, 1963). This assumption is plausible for circle size because of the small range,

Table 5

Rank and Partial Correlations Between Estimated Similarity and Different Criteria for 42 Os. $(C=$ circle size, $A=$ angle,$D=$ difference, $R=$ ratio, $H=$ from horizontal, $V=$ from vertical. $)$ The decimal points are left out.

\begin{tabular}{|c|c|c|c|c|c|c|c|c|c|}
\hline \multirow[b]{2}{*}{ O No. } & \multicolumn{5}{|c|}{ Rank Correlations } & \multicolumn{4}{|c|}{ Partial Correlations } \\
\hline & $\mathrm{CD}$ & $\mathrm{CR}$ & $\mathbf{A D}$ & ARH & ARV & $\mathrm{C}$ & & A & $\mathbf{A}$ \\
\hline 1 & -48 & -54 & 68 & 65 & 65 & (18 & D) & 57 & $\mathrm{D}$ \\
\hline 2 & 94 & 93 & -75 & -75 & -72 & 88 & D & (17 & RV) \\
\hline 3 & 83 & 83 & -61 & -61 & -59 & 74 & $\mathbf{R}$ & (25 & RV) \\
\hline 4 & -71 & -73 & 87 & 80 & 84 & $(-01$ & D) & 70 & D \\
\hline 5 & 38 & 45 & -17 & -19 & -17 & 55 & $\mathbf{R}$ & 39 & D \\
\hline 6 & 82 & 84 & -66 & -69 & -65 & 70 & $\mathbf{R}$ & (09 & $\mathrm{RV}$ \\
\hline 7 & 88 & 90 & -79 & -73 & -81 & 78 & $\mathbf{R}$ & $(02$ & RH) \\
\hline 8 & 07 & 12 & 14 & 14 & 08 & 41 & $\mathbf{R}$ & 42 & $\mathrm{D}$ \\
\hline 9 & 33 & 27 & -11 & -18 & -08 & 45 & $\mathrm{D}$ & 34 & RV \\
\hline 10 & -71 & -71 & 90 & 90 & 83 & (15 & D) & 80 & D \\
\hline 11 & -57 & -56 & 37 & 34 & 43 & -53 & $\mathrm{D}$ & -24 & RH \\
\hline 12 & -23 & -19 & 41 & 35 & 39 & 29 & $\mathbf{R}$ & 46 & $\mathrm{D}$ \\
\hline 13 & -56 & -58 & 79 & 71 & 80 & $(24$ & D) & 71 & RV \\
\hline 17 & 92 & 94 & -81 & -78 & -81 & 85 & $\mathbf{R}$ & $(-06$ & $\mathbf{R H )}$ \\
\hline 15 & 84 & 84 & -69 & -63 & -72 & 72 & $\mathrm{D}$ & $(14$ & $\mathbf{R H )}$ \\
\hline 16 & 75 & 74 & -60 & -57 & -58 & 60 & $\mathrm{D}$ & $(09$ & RH) \\
\hline 17 & 95 & 96 & -82 & -81 & -80 & 90 & $\mathbf{R}$ & $(-13$ & RV) \\
\hline 18 & -14 & -17 & 23 & 18 & 23 & (09 & D) & (21) & RV) \\
\hline 19 & 26 & 23 & -02 & -05 & -10 & 42 & $\mathbf{D}$ & 34 & $\mathrm{D}$ \\
\hline 20 & 48 & 46 & -23 & -23 & -22 & 53 & $\mathrm{D}$ & 32 & RV \\
\hline 21 & 86 & 88 & -72 & -70 & -71 & 76 & $\mathbf{R}$ & $(07$ & $\mathbf{R H )}$ \\
\hline 22 & 91 & 92 & -76 & -74 & -76 & 82 & $\mathbf{R}$ & (06 & RH) \\
\hline 23 & -11 & -10 & 28 & 28 & 23 & 24 & $\mathbf{R}$ & 35 & D \\
\hline 24 & 12 & 14 & 11 & 11 & 03 & 41 & $\mathbf{R}$ & 41 & D \\
\hline 25 & 72 & 70 & -42 & -41 & -48 & 72 & D & 42 & D \\
\hline 26 & 59 & 55 & -28 & -32 & -29 & 65 & D & 44 & D \\
\hline 27 & 75 & 74 & -53 & -52 & -53 & 65 & D & $(20$ & RH) \\
\hline 28 & 80 & 72 & -51 & -51 & -53 & 78 & D & 43 & D \\
\hline 29 & 68 & 63 & -40 & -42 & -41 & 67 & $\mathrm{D}$ & 38 & D \\
\hline 30 & 49 & 46 & -21 & -28 & -20 & 56 & $\bar{D}$ & 37 & RV \\
\hline 31 & 24 & 23 & -14 & -13 & -15 & (22 & D) & $(10$ & RH) \\
\hline 32 & -04 & -04 & 37 & 34 & 34 & 49 & $\mathbf{R}$ & 59 & D \\
\hline 33 & 95 & 95 & -77 & -75 & -76 & 89 & D & $(10$ & RH) \\
\hline 34 & 31 & 25 & -06 & -08 & -06 & 47 & D & 37 & D \\
\hline 35 & 39 & 40 & -19 & -15 & -21 & 49 & $\mathbf{R}$ & 33 & $\mathbf{R H}$ \\
\hline 36 & 86 & 82 & -63 & -62 & -61 & 79 & D & $(28$ & RV) \\
\hline 37 & -21 & -16 & 33 & 33 & 26 & 20 & $\mathbf{R}$ & 34 & D \\
\hline 38 & -71 & -74 & 83 & 80 & 79 & $(-10$ & D) & 62 & RH \\
\hline 39 & 89 & 88 & -78 & -78 & -73 & 73 & D & $(-08$ & RV) \\
\hline 40 & 03 & 03 & 25 & 20 & 30 & 49 & $\mathbf{R}$ & 55 & RV \\
\hline 41 & 12 & 18 & 19 & 15 & 17 & 61 & $\mathbf{R}$ & 61 & D \\
\hline 42 & 50 & 49 & -13 & -16 & -14 & 69 & D & 56 & D \\
\hline
\end{tabular}

and for angle because of the almost linear psychophysical function (Goude, 1962). For the ratio criteria this is not a prerequisite.] The rank correlations were computed, following a recommendation by Hays (1963), as product moment correlations between ranks, corrected by Kendall's rank correlation. Table 5 gives these correlations.

These rank correlations do not contain much information in themselves. It can only be seen that different Os attend most to different criteria, but their distribution of attention between the two dimensions is difficult to judge. However, making use of the fact that the correlations between the two dimensions are about -.8 (see Table 4) for the figure pairs in Experiment 1, the partial correlation with one of the dimensions, keeping the other constant, and vice versa, tells about the attention paid to the two dimensions separately. Since there are two criteria for circle size and three criteria for angle, the following procedure was adopted.

The partial correlations of the Os' similarity estimates with each criterion of one dimension were computed, keeping all the criteria of the other dimension constant in turn. For instance, the partial correlation of the similarity estimates with the ratio of circle size was computed, keeping the angle difference constant. Accordingly, 12 partial correlations were computed for every $O$. From these 12 , the highest was picked together with the corresponding correlation in the other dimension, the latter almost always being the highest there. For example, if the highest partial correlation was with the angle ratio from vertical, with circle size difference constant, the other was circle size difference with angle ratio from vertical constant. Table 5 gives these correlations, too. Values within parentheses were considered so small as to be negligible. The letters indicate the criterion used by the $O$. Because the basis of the partial correlations are rank correlations, no statistical test as to significance was carried out. The results are summarized in Table 6 . Three Os do not appear in Table 6. Two of them, Os 18 and 31, had such low correlations in both dimensions that they either switched continuously between different criteria or made only random judgments, and one, 011 , having negative partial correlations with both dimensions, seems to have estimated dissimilarity (with circle size difference as the main and angle ratio from the horizontal as the second criterion).

Two criteria, which were in good agreement, were used to determine whether an $\mathrm{O}$ attended to both dimensions or not: (1) Both partial correlations had to 
Table 6

Number of Os Using Different Criteria for Their Similarity Judgments. $(C=$ circle size, $\mathbf{A}=$ angle,$\quad \mathbf{C}+\mathbf{A}=$ both dimensions, $D=$ difference, $R=$ ratio, $H=$ angle from horizontal, $\mathrm{V}=$ angle from vertical.)

\begin{tabular}{lrrrrrr}
\hline & CD & CR & AD & ARH & ARV Total \\
\hline C + A & 10 & 10 & 15 & 1 & 4 & 20 \\
C & 7 & 7 & & & & 14 \\
A & & & 3 & 1 & 1 & 5 \\
Total & 17 & 17 & 18 & 2 & 5 & \\
\hline
\end{tabular}

exceed (or at least not lie below) the corresponding rank correlations, and (2) the square of the ratio of the higher to the lower partial correlation must not exceed 3.5. The latter measure was chosen as a crude estimation of the ratio of the variances accounted for by the two dimensions, and the particular value, because in the distribution of this criterion over Os there was a gap between 3.3 and 7.9 .

In the Os attending to one dimension only, the partial correlation with his main criterion typically lies below the rank correlation. This may be taken to indicate that the $O$ "made use" of the negative correlation between the dimensions in his judgments and in this sense attended to the other dimension, too.

The partial correlations were transformed to Fisher's $z$ and are plotted ( $z$ for circle size vs $z$ for angle) in Fig. 4 (O 11, with his negative correlations, is left out). The correlation between the two $\mathrm{z}$ values over $\mathrm{Os}$ is -.65 , indicating that, by and large, the more an $\mathrm{O}$ attends to one dimension, the less he attends to the other. (Note that this finding is not trivial. For a correlation of -.8 between criteria, and correlations between similarity judgments and criteria of .316 , both partial correlations would equal unity. The distance from the origin for a point in Fig. 4 may be regarded as an indicator of the total attention.)

In an attempt to obtain more information, a component analysis of the similarity estimates according to Tucker and Messick (1963) was carried out. The values of the first eigenroots were $10216069,198622,35584,29279$, 21420 . From these values it can be seen that probably only the first two factors contain any information. The first factor agreed with the Os' mean judgments, as expected. The second factor seems to describe the Os' attention to angle compared to circle size. In Fig. 5 , the

Fig. 5. Differences between $z$ values of partial correlations with angle and circle size, respectively, vs Factor II from factor analy sis of similarity estimates according to Tucker \& Messick (1963) for 42 Os. difference, $z_{\text {angle }}-z_{\text {circle, }}$ is piotted $v s$ Factor II. There is a clear, though far from perfect, relation. The correlation between the two variables amounts to .89 . No interpretation could be found for the third factor.

\section{Discussion}

The partial correlations given in Table 5 make it possible not only to distinguish between one-dimension attenders and two-dimension attenders but also to estimate relative degree of attention between the two dimensions in the latter. The question remains, however, whether an $O$ weighted the dimensions when making a judgment or whether he switched attention from one dimension to the other. The correlations mentioned above inform only as to the overall attention.

A rough method of determining the consistency of an O's similarity judgments as to choice of criterion is to look at the distribution of high ranks (e.g., $\geqslant 50$ ) between circle size matching, angle matching, and attention to both. Table 7 gives this distribution. It will be seen that of the 20 two-dimension attenders, only 3 (Os 23, 37, and 42) never attended to one dimension only, whereas 5 (Os 25, 28, 29, 35 , and 40) never attended to both dimensions simultaneously, all according to the criterion mentioned above.

The conclusion is that most Os are unable to carry out similarity judgments following the same criterion for all

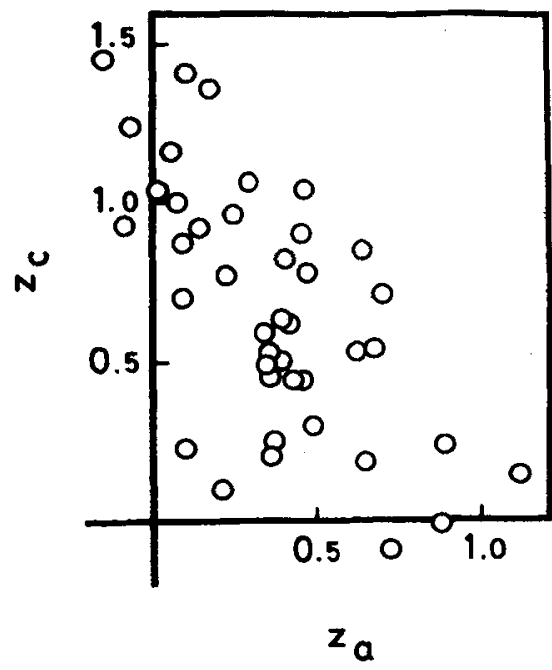

Fig. 4. 2 values for partial correlations for circle size vs angle for 41 Os.

stimulus pairs when different dimensions are clearly discernible. Of the $42 \mathrm{Os}$ in Experiment 1, 1 estimated dissimilarity instead of similarity, 1 made quite haphazard judgments, 23 switched between different criteria, 12 attended to circle size exclusively, 1 to angle exclusively, 3 to both dimensions consistently, and 1 is difficult to classify since he differentiated very little between figure pairs in his estimates.

As to the classification of Os according to use of difference or ratio as criterion, a

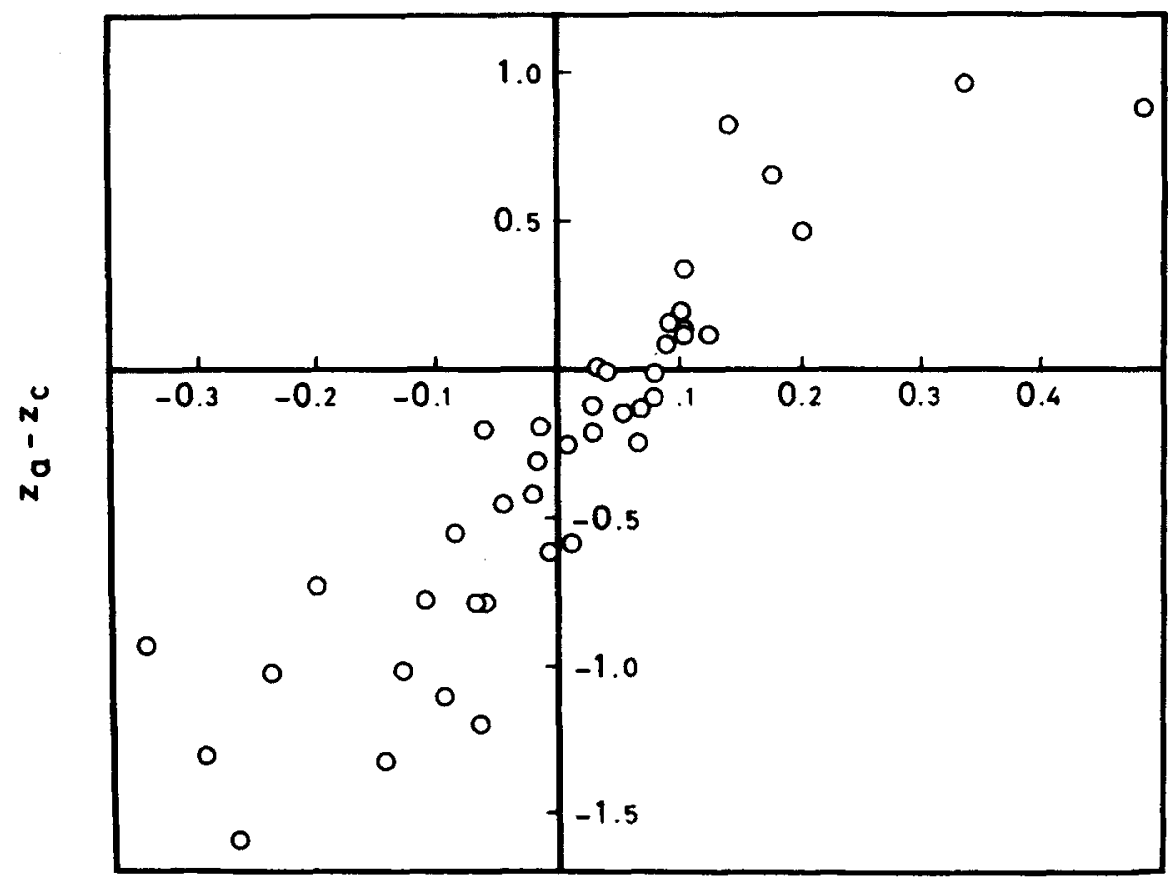

Factor II 
Table 7

Number of Ranks of 50 or Greater for Circle Size Matching and Angle Matching (Figure Pairs C, D, E, and K, L, M, Depending on Series) and for Distributed Attention (Figure Pairs G, H, I), Together with Type of $\mathbf{O}$ According to Partial Correlations.

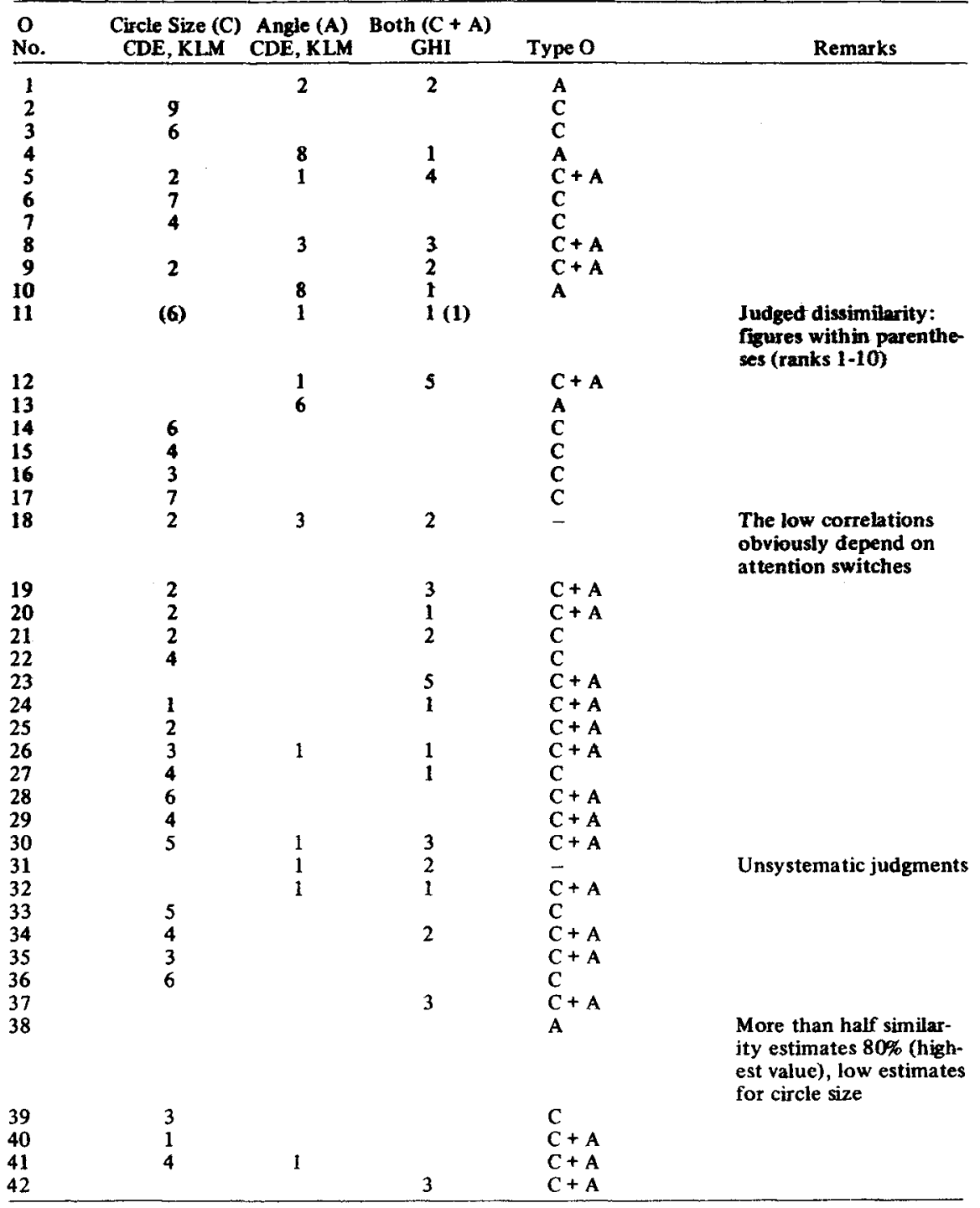

closer analysis does not seem worthwhile, since the high correlations between these criteria make the classifications, as given in Table 6, unreliable. Taking the distributions in Table 6 for what they are, it seems that different Os make use of different criteria as to ratio and difference. Thus it cannot be taken for granted that what is closest between two stimuli in the sense of physical difference is closest subjectively, too.

\section{SUMMARY AND CONCLUSIONS}

Forty-two Os rated similarity of stimulus pairs with two clearly discernible dimensions (a circular rim of varying size with a spoke of varying inclination). This experiment differed from Shepard's (1964) mainly in that Shepard had his Os pick the stimulus pair with the greatest similarity. In agreement with Shepard's results, it was found that $O$ s could be classified in to three groups, those attending only to circular size, those attending only to angle, and those attending to both dimensions. The proportion of Os attending to both dimensions was lower in the present than in Shepard's experiment. The negative correlations exhibited between measures of the two dimensions for the present stimulus series allowed the computation of partial correlations of an $O$ 's similarity estimations with any one dimension and thus yielded a measure of relative degree of attention. Attention to circle size correlated negatively with attention to angle. The partial correlations are only an overall measure, however, and a closer scrutiny of the data demonstrated that almost half of the Os used different similarity criteria during the course of the experiment, and most of the rest attended to only one dimension. Only three Os attended consistently to both dimensions.

Shepard conchudes from his experiment that no metric exists for group data. This contention seems meaningful to us only when an unambiguous isosimilarity contour $^{5}$ can be determined, the properties of which inform as to possible violations of conditions for metricity. This requirement is not met for the group as a whole, since the application of different criterin by different Os necessarily results in a wide band rather than in a contour. (It is, however, met by the subgroup of matchers, and for them one can say that no metric exists.)

Further, Shepard claims that for individual Os "in any one state of attention" some Minkowski metric might be applicable. To make this statement empirically meaningful beyond pure definition would require a stimulus-contingent system in an $O$ 's responses. Such a system can only be found by a detailed study of an O's responses over replications, which was not done by Shepard or us. ${ }^{6}$ If, on the other hand, an O's attention shifts independently of the stimulus shown, as our date indicate, if may be a better description to say that he is unable to carry out his task.

Eisler (1960) made a distinction between different dimensions and different bodies of dimensions and concluded: "... it seems reasonable to assume that the concept 'similarity' can be meaningfully applied to two percepts belonging to the same dimension or to the same body of dimensions, but not to two percepts belonging to different bodies of dimensions. An observer instructed to estimate the similarity of e.g., two differently colored weights, is supposed to ask: In what respect?" And that is exactly what our (and Shepard's) Os should have done instead of trying to comply with an impossible instruction.

\section{REFERENCES}

EISLER, H. Similarity in the continuum of heaviness with some methodological and theoretical considerations. Scandinavian Journal of Psychology, 1960, 1, 69-81.

EKMAN, G., \& SJÖBERG, L. Scaling. Annual Review of Psychology, 1965, 16, 451-474.

GOUDE, G. On fundamental measurement in psychology. Uppsala: Almqvist \& Wiksell, 1962.

HAYMAN, R., \& WELL, A. Judgments of similarity and spatial models. Perception \& Psychophysics, 1967, 2, 233-248. 
HAYMAN, R., \& WELL, A. Perceptual separability and spatial models. Perception \& Psychophysics, 1968, 3, 161-165.

HAYS, W. L. Statistics for psychologists. New York: Holt, 1963.

SHEPARD, R. N. The analysis of proximities: Multidimensional scaling with an unknown distance function. I, II. Psychometrika, 1962, 27, 125-140, 219-246.

SHEPARD; R. N. Attention and the metric structure of the stimulus space. Journal of Mathematical Psychology, 1964, 1, 54-87.

SUPPES, P., \& ZINNES, J. Basic measurement theory. In R. D. Luce, R. R. Bush, and E. H. Galanter (Eds.); Handbook of mathematical psychology. Vol. 1. New York: Wiley, 1963. Pp. 1.76 .

TUCKER, L. R., \& MESSICK, S. An individual difference model for multidimensional scaling. Psychometrika, 1963, 28, 333-367.

\section{NOTES}

1. Research supported by grants from the Swedish Social Science Research Council.

2. Address: Psychological Laboratories University of Stockholm, Stockholm, Sweden.

3. Accordingly, the standard stimulus always consisted of a circle with a diameter of $1.905 \mathrm{~cm}$ and a spoke with an inclination of $45.0 \mathrm{deg}$. The corresponding values of circle size and spoke inclination for the comparison stimuli are found as the coordinates of the lettered points. $A$ tangential series comprises 15 comparison stimuli and is denoted by the number where the tangent touches the circle.

4. This view is probably a consequence of the distance model for similarity (Shepard, 1962). For unidimensional continua, however, the ratio rather than the difference between subjective values seems to be the relevant variable for similarity (Eisler, 1960). This may also be the case for multidimensional similarity (the content model, see Ekman \& Sjöberg, 1965, for a comparison). In the investigation reported here no stand is taken as to the two models.

5. An isosimilarity contour is the locus of all stimuli that have any prescribed degree of similarity to any prescribed standard stimulus.

6. The production method used by Hy man and Well $(1967,1968)$ for the same stimulus material made use of replications. The high correlations found by them indicate that, for their method. attention shifts. if any, were systematic.

(Accepted for publication March 3, 1970.) 